\title{
Adaptación del ĺndice de Gini a investigaciones sociológicas sobre la educación
}

\author{
Adaptation of The Gini Index to Sociological Research on Education
}

Francesc Jesús Hernàndez i Dobon, Uyguaciara Veloso Castelo Branco y Paulo Hideo Nakamura'

\section{Resumen}

El artículo propone una adaptación del Índice (o Coeficiente) de Gini, habitual para medir la desigualdad en la economía y otras ciencias, a investigaciones sociológicas de la educación. Explica detalladamente el funcionamiento del índice, su mecánica de cálculo, y sus ventajas y expone tres cálculos, con diferentes procedimientos de cálculo, aplicados al ámbito educativo: uno relativo a la inversión pública en educación superior, otro referente a los ajustes de las trayectorias universitarias y el último relacionado con las competencias lingüísticas.

\section{Palabras clave}

Índice de Gini, Coeficiente de Gini, tasa de fluidez, desigualdad, indicadores educativos.

\section{Abstract}

The article proposes an adaptation of the Gini Index (or Coefficient), customary to measure inequality in the economy and other sciences, to sociological research of education. It explains in detail the operation of the index, its calculation mechanics, and its advantages and exposes three calculations, with different calculation procedures, applied to the educational field: one related to public investment in higher education, another referring to the adjustments of university trajectories and the last one related to language skills.

\section{Keywords}

Gini Index, Gini Coefficient, fluency rate, Inequality, educational indicators.

\section{Cómo citar/Citation}

Hernàndez i Dobon, Francesc Jesús; Castelo Branco, Uyguaciara Veloso; Nakamura, Paulo Hideo (2020). Adaptación del Índice de Gini a investigaciones sociológicas sobre la educación. Revista de Sociología de la Educación-RASE, 13 (1), 52-62. http://dx.doi.org/10.7203/ RASE.13.1.16283.

1 Francesc Jesús Hernàndez i Dobon, Universitat de València, francesc.j.hernandez@uv.es; Uyguaciara Veloso Castelo Branco, Universidad Federal de Paraíba, uyguaciara@gmail.com y Paulo Hideo Nakamura, Universidad Federal de Paraíba,nkm.paulo@gmail.com. 


\section{Introducción}

No deja de ser curioso que, teniendo la sociología como uno de sus objetos más destacados (si no, su objeto por antonomasia) la desigualdad social, utilice escasamente los instrumentos matemáticos que, en otras disciplinas, y paradigmáticamente en la economía, se usan para evaluar la desigualdad socioeconómica. Este hecho puede deberse a una pluralidad de factores, entre los que se podrían apuntar la restricción del análisis matemático al uso del instrumental estadístico o la proliferación de investigaciones exclusivamente cualitativas (debidas en muchos casos a la escasez de medios o a aparatos teóricos aparentemente antagónicos con los procedimientos cuantitativos).

En tiempos recientes, los llamados «números de la educación» se han convertido en una herramienta importante para el empleo de los recursos en educación y, consiguientemente, para los análisis políticos y financieros. No sin razón, los indicadores han aumentado en cantidad y calidad en todo el mundo. En una relevante revisión del tema, Bandura (2006) ha identificado más de ciento sesenta indicadores compuestos, que son utilizados por diversos organismos internacionales para medir o comparar el desempeño de países interna o externamente (OCDE, 2008: 3). Para una mejor comprensión de la noción de indicador, adoptamos la definición siguiente:

"Un indicador es una estadística que mide nuestro bienestar colectivo. Un verdadero indicador mide la salud de un sistema, sea económico, de empleo, de servicios médicos o educativos [...] A diferencia de otras estadísticas, un indicador debe ser relevante para la toma de decisiones, en función de ciertos problemas; debe ofrecer información sobre un rasgo significativo del sistema al que se refiere; $y$ generalmente incluye algún estándar contra el cual pueda juagarse si hay progreso o retroceso». (Special Study Panel on Indicators 1991: 12).

No está de más recordar que el arsenal matemático no se restringe a los instrumentos estadísticos o probabilísticos. Hemos escrito este artículo precisamente para reivindicar aproximaciones matemáticas, digamos, más ambiciosas y mostrar su virtualidad en la investigación. Tal es el caso del Índice o Coeficiente de Gini, elaborado por el estadístico, matemático y sociólogo italiano Corrado Gini y es una medida estandarizadaque se puede utilizar para medir cualquier forma de distribución desigual, como por ejemplo, la renta, por la ciencia económica (OCDE 2011: 21-45)².

El Índice de Gini (en adelante, IG) es un valor numérico (entre 0 y 1 ) que expresa la magnitud de una relación desigualitaria. Se denomina Coeficiente de Gini (CG) al resultado de multiplicar el IG por 100. En su formulación original, el IG ofrece una medida de desigualdad económica en una sociedad y un tiempo determinados (cf. INE 2019). Se usa en instituciones financieras y estadísticas, aunque, en estos casos, se suele preferir su presentación como CG (cf. Banco Mundial 2019). Tanto el IG como el CG han sido utilizados en otros ámbitos científicos, tan heterogéneos como la investigación sobre la pesca (Pérez et al., 2005), el estudio de la depresión (Maldonado et al., 2007), la medida de la lluvia (Benhamrouche y Martín 2012) o la distribución de les flores (Grajales et al., 2016), pero muy escasamente en investigaciones sociológicas sobre la educación. En algunas ocasiones se han establecido relaciones entre rendimientos educativos y valores del IG, calculado este al modo habitual (Martínez Rizo, 2012), pero no se ha explotado su potencialidad en la propia investigación socioeducativa. En este artículo se explicará esa

\footnotetext{
2 Aunque existen programas matemáticos especializados, en este caso nos hemos limitado también a procedimientos que se pueden realizar con cualquier hoja de cálculo habitual (como Excel, Numbers, Calc o la integrada en Google Apps) o las calculadoras científicas que tienen incorporados los ordenadores o los teléfonos portátiles. Además, para favorecer su lectura, hemos descargado la exposición de farragosas demostraciones matemáticas, que naturalmente podríamos proporcionar a los lectores interesados.
} 
potencialidad y se aportarán algunos casos de investigaciones propias. Nuestro objetivo al extendernos con las consideraciones matemáticas es, precisamente, a partir de la ilustración de su funcionamiento, animar otras aplicaciones originales en el ámbito educativo.

El IG se presenta como una alternativa a otros modos de medir la desigualdad. Para establecer la magnitud de una relación desigualitaria también es frecuente la utilización de proporciones entre cuantiles (quintiles, deciles, etc.). Así, por ejemplo, Eurostat mide la desigualdad de los países miembros de la Unión Europea con la proporción de ingresos entre el quintil superior y el inferior (lo que abrevia: S80/S20, cf. Eurostat 2019). La OCDE también ofrece proporciones semejantes en su estimación de la desigualdad económica. Adviértase que en estos casos se ponen en relación dos variables (a saber, la población y los ingresos), pero solo se usa una muestra (dos quintiles, dos deciles, etc., de la población, cf. también OCDE 2019). En el caso del IG, la relación establecida se refiere a todo el universo de las variables.

Introduciremos ahora algunas consideraciones sobre la dinámica del cálculo del IG, tal vez arduas pero necesarias para la comprensión de los casos educativos.

\section{Consideraciones metodológicas}

Como en los casos anteriores, el IG opera con variables numéricas y una variable $(x)$ está ordenada en función de la otra $(y)$. Por ejemplo: la población se ordena respecto de sus ingresos. Pero, y esta es una diferencia notable, en el caso del IG se utilizan los porcentajes acumulados, lo que, como se verá más adelante, simplifica considerablemente el cálculo. De este modo, la relación entre ambas variables cumple dos requisitos que no resultan triviales: a) al $0 \%$ de $x$ le corresponde necesariamente el $0 \%$ de $y$; y b) al $100 \%$ de y le corresponde necesariamente el 100\% de $x$. En nuestro ejemplo anterior, resulta obvio que el $0 \%$ de la población acumula el $0 \%$ de los ingresos y que el $100 \%$ de los ingresos es lo acumulado por el $100 \%$ de la población.

Ahora bien, no resulta una cuestión menor determinar en otros casos qué variable designaremos como $x$ y qué variable designemos como $y$. En general, se puede establecer el criterio siguiente: el porcentaje de $y$ ha de ser igual o inferior al porcentaje de $x$ correspondiente. A continuación, se explica esta afirmación. Tomemos un ejemplo de la sociolingüística. Imaginemos en un contexto plurilingüe que hay individuos con la capacidad (competencia) de hablar (uso) más de una lengua. Es fácil deducir que la tasa de uso de una lengua determinada será igual o inferior a la tasa de competencia. En este caso, la tasa de competencia será la variable $x$ y la tasa de uso la variable $y$.

Si representamos los valores de $x$ e $y$ en unos ejes cartesianos habituales, el conjunto de los pares de valores definirán una línea (no entraremos todavía a tratar qué tipo de línea), que denominaremos $L$, y que cumple dos condiciones: a) necesariamente pasa por los puntos $(0,0)$ y $(1,1)$, por las condiciones expresadas anteriormente de que al valor $0 \%$ de $x$ les corresponde $0 \%$ de $y$, y que al $100 \%$ de $y$ le corresponde el $100 \%$ de $\mathrm{x}$; y b) los puntos $(x, y)$ se colocan por debajo de la diagonal que une los puntos $(0,0)$ y $(1,1)$, lo cual es lógico, porque, si la relación fuera igualitaria, los puntos coincidirían con la diagonal, toda vez que la proporción acumulada de una variable coincidiría con la proporción acumulada de la otra.

Pues bien, el IG se define precisamente como la proporción de la superficie acotada por el segmento $(0,0)$ y $(1,1)$, mencionado, y la línea $L$ (superficie que denominaremos $G$ ) respecto de la superficie por debajo de la diagonal, es decir, respecto del triángulo formado por el segmento mencionado y el punto 
$(0,1)$. Es decir, se trata de establece qué tan grande es $G$ por lo que tenemos que tomar como referencia alguna parte del rectángulo para establecer una relación. En el Gráfico 1 podrá apreciarse mejor lo dicho.

\section{Gráfico 1}

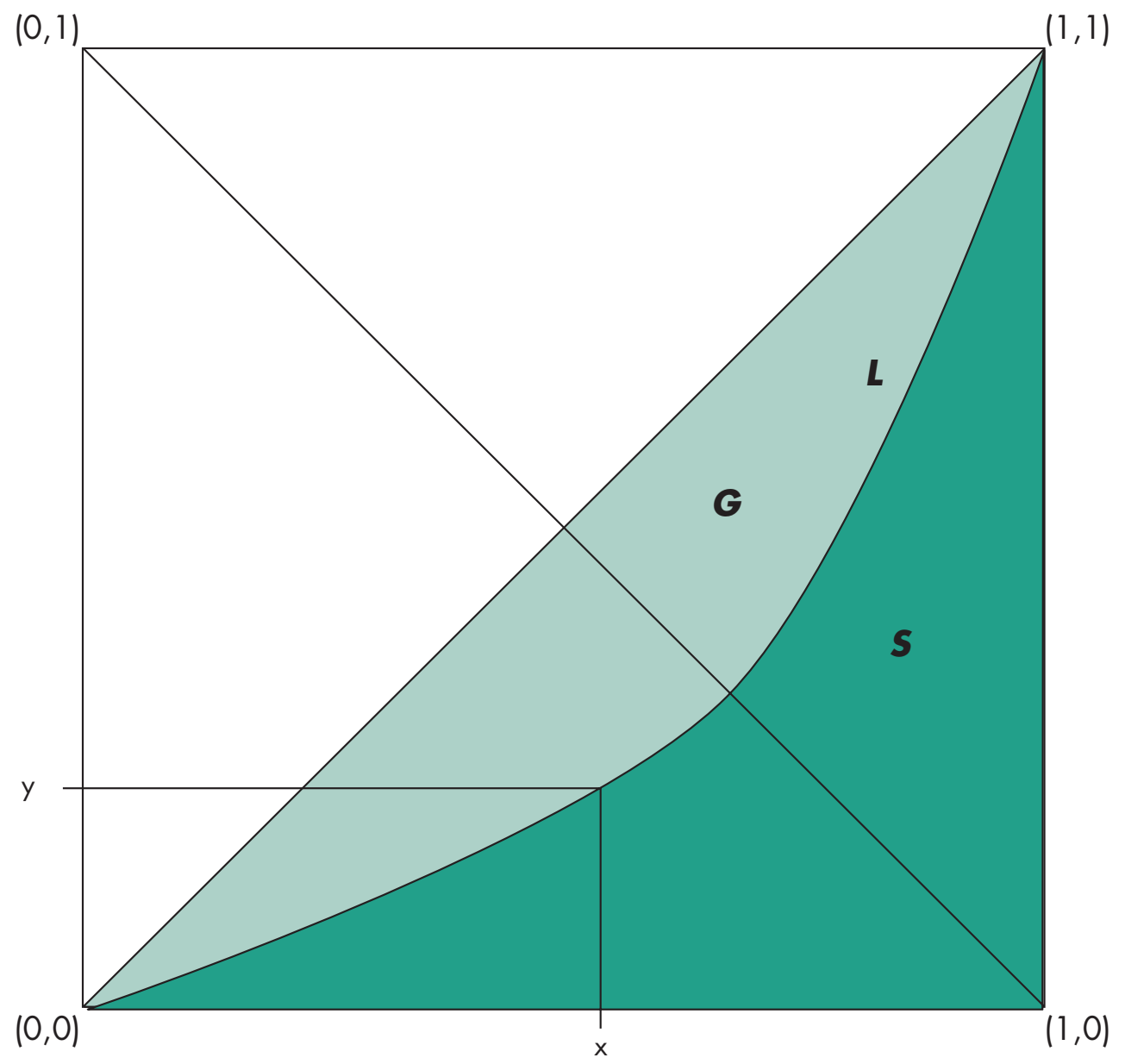

Como los ejes corresponden a porcentajes acumulados, la superficie del triángulo equivale a $1 / 2$ unidades. Si denominamos $S$ a la superficie por debajo de la línea $L$, tendremos, por tanto:

$$
\text { IG }=\frac{G}{G+S}=\frac{G}{\frac{1}{2}}=2 G
$$

Ahora bien, en términos geométricos resulta más fácil calcular S, por lo que el procedimiento más sencillo parte de:

$$
G=\frac{1}{2}-S
$$

Y, por tanto:

$$
\mathrm{IG}=2 G=2\left(\frac{1}{2}-S\right)=1-2 S
$$

Tenemos que acometer ahora la cuestión del tipo de línea que une los puntos $(0,0),(x, y)$ y $(1,1)$. Para simplificar esta cuestión, la reduciremos a dos casos, en orden creciente de complejidad de cálculo de $S$, y comentaremos posteriormente dos casos más.

\section{Caso $1^{\circ}$. L és una línea recta quebrada}

Esto sucede, por ejemplo, si disponemos de los valores de $(x, y)$ por intervalos. Se produce este caso, por ejemplo, cuando se trata de datos de cuantiles (quintiles, deciles, etc.). Entonces, la línea recta que- 
brada define trapezoides con el eje $x$, cuya superficie se puede calcular fácilmente, porque en definitiva se trata de figuras compuestas de rectángulos más triángulos rectángulos, que comparten la misma base. También puede calcularse $S$ mediante el producto de los intérvalos de $\chi$ (base de cada trapezoide) por la semisuma de los valores de $y$ (alturas), es decir, recurriendo a las marcas de clase.

Naturalmente, se puede trazar una línea de tendencia curva por los valores de $(x, y)$ y entonces estaríamos en el caso $2^{\circ}$.

\section{Caso $2^{\circ}$. L es una línea curva.}

En ese caso, el área de $S$ se puede calcular a partir de la integral definida de la función de la curva. Se trata del problema clásico del área bajo curva. Y es en este punto cuando se advierte la ventaja de utilizar porcentajes acumulados, por cuanto, en este caso, la integral se define entre los valores 0 y 1 , lo que simplifica extraordinariamente los cálculos. Si la función de la curva está definida por un polinomio de grado $n$, con la forma:

$$
y=a x^{n}+b x^{n-1}+\cdots+p x+q
$$

entonces el área de $S$ se puede calcular fácilmente, porque:

$$
S=\frac{a}{n+1}+\frac{b}{n}+\cdots+\frac{p}{2}+q
$$

Obsérvese como el problema clásico del área bajo curva, con integrales definidas, se reduce en este caso a una suma de fracciones cuyos denominadores son números naturales.

Además de estos dos casos, hay otros dos cuya singularidad merece ser comentada, a saber, que la línea $L$ sea una recta o que dispongamos solo de un punto $(x i, y i)$.

\section{Caso $3^{\circ}$. Si L es una línea recta}

Entonces resulta trivial que ha de coincidir con la diagonal, lo que, como ya hemos comentado, acaece cuando hay igualdad plena. No merece más comentario este caso.

\section{Caso $4^{\circ}$. Resulta sumamente interesante el caso en el que dispongamos de un solo punto (xi, yi).}

En realidad, siempre se dispone de tres puntos, porque hay que añadir $(0,0)$ y $(1,1)$. En este caso, lo más acertado entendemos que sería trazar una curva que pasara por los tres puntos y proceder al cálculo del área bajo curva, etc. Se puede recurrir a una hoja de cálculo, representar los puntos en un diagrama de dispersión y trazar una línea de tendencia curva que pase por los tres puntos. Después, colocando el cursor en un punto, pedirle al programa que nos devuelva la ecuación de la curva y seguir el procedimiento comentado ya. El problema se plantea cuando la curva trazada se aleja excesivamente de los puntos, presenta puntos de inflexión (es decir, segmentos crecientes y segmentos decrecientes) o, incluso, corta el eje $x$ e invade, por decirlo así, el cuarto cuadrante (el área con valores de x positivos, pero con valores de y negativos o en términos más prosaicos, la línea queda por debajo del segmento del eje x que nos interesa). Para solucionar estos problemas hemos desarrollado dos procedimientos, según optemos por una curva parabólica o exponencial.

El primer caso es de la curva parabólica, es decir, aquella curva que añade la condición de simetría respecto de la diagonal que une los puntos $(1,0)$ y $(0,1)$. En este caso, por la condición de simetría contamos también con el punto $(1-y i, 1-x i)$, que es el simétrico de $(x i, y i)$. Para resolver los problemas se- 
ñalados, podemos simplificar el procedimiento de cálculo procediendo a un desplazamiento y un giro de los ejes de coordenadas (cf. Pontriaguin, 2011); operamos un desplazamiento de una unidad hacia la derecha y un giro de $45^{\circ}$ hacia la izquierda). No es preciso seguir aquí todo este problema geométrico (que recurre a la trigonometría básica). Aportaremos solo su conclusión y el procedimiento de cálculo. En este caso, basta cargar en una hoja de cálculo los nuevos valores de $x$ e $y$, representarlos en un gráfico de dispersión, solicitar al programa que trace una línea de tendencia polinómica (como siempre, poniendo el cursor sobre uno de los tres puntos, etc.) y que nos devuelva la ecuación de su curva; a continuación, se calcula el IG con una sencilla fórmula que indicamos a continuación. Si disponemos del par de valores $\left(x_{i}, y_{i}\right)$, cargaremos en la hoja la Tabla I:

Tabla I. Valores de $x$ e $y$ a partir de $x_{i}$ e $y_{i}$

\begin{tabular}{cc}
\hline$x$ & $\frac{\sqrt{2}}{2}$ \\
\hline$-\frac{\sqrt{ } 2}{2}$ & $\frac{\sqrt{ } 2}{2}$ \\
\hline$\frac{\sqrt{ } 2}{2}$ & $\frac{\left(y_{i}+1-x_{i}\right) \sqrt{ } 2}{2}$ \\
\hline$\frac{\left(x_{i}-1+y_{i}\right) \sqrt{ } 2}{2}$ & $y^{2}$ \\
\hline
\end{tabular}

Recuérdese que $\frac{\sqrt{ } 2}{2}=0,70710678$, lo que simplifica bastante las fórmulas de la tabla anterior. Naturalmente, las dos primeras filas corresponden a los puntos $(1,0)$ y $(0,1)$ después de proceder al desplazamiento y el giro de los ejes cartesianos. Cuando, a partir de los nuevos valores indicados, tengamos la ecuación de la línea de tendencia (polinómica), con la forma: $y=a x^{2}+b x+c$, entonces calcularemos IG con la fórmula siguiente (economizamos al lector la deducción correspondiente):

$$
I G=2-\frac{\sqrt{ } 2}{3} a-b-2 \sqrt{ } 2 c
$$

Naturalmente este método es bastante más ágil que el cálculo de la ecuación de la curva mediante sistemas de ecuaciones, caso de disponer solo de programas como las hojas de cálculos habituales.

En el caso de la curva exponencial, esto es, de aquella curva que no está afectada por la condición de simetría respecto de la diagonal que une los puntos $(1,0)$ y $(0,1)$, podemos utilizar una ecuación del tipo: $y=\left(2^{x}-1\right)^{a}$. Aunque se podrían utilizar múltiples ecuaciones, hemos optado por la indicada porque es una de las más simples que cumple las dos condiciones establecidas, a saber: si $x=0$ (o $0 \%$, entonces $y=0$, y si $y=1$, necesariamente $x=1$. Hay otras ecuaciones que también cumplen esta condición, por ejemplo:

$$
y=k\left(e^{a x}-1\right)
$$

Pero que no hemos desarrollado todavía.

En el caso propuesto, a saber: $\mathrm{y}=\left(2^{x}-1\right)^{a}$, para un par de valores $\left(x_{i}, y_{i}\right)$, podemos deducir que:

$$
a=\frac{\log y_{i}}{\log \left(2^{x_{i}}-1\right)}
$$

Y entonces, utilizando nuestra hoja de cálculo, simplemente asignaremos valores hipotéticos de $x$ (por ejemplo, cada 0,05 o cada 0,1 puntos, entre 0 y 1) a la ecuación resultante (donde ya tenemos el valor 
de a) y obtendremos los y correspondientes pares de valores, los representaremos en un diagrama, trazaremos la curva, le pediremos al programa que nos devuelva su ecuación polinómica, averiguaremos $S$ y resolveremos el cálculo del IG de la manera indicada anteriormente, a saber: IG $=1-2 S$. Naturalmente, a mayor número de puntos y mayor grado de la ecuación polinómica (algunas hojas de cálculo permiten ecuaciones de grado 6), podemos suponer que la precisión será mayor. Con un poco de práctica en el uso de las funciones de las celdas de la hoja de cálculo, este procedimiento resulta mucho más sencillo de lo que se puede suponer contemplando las fórmulas con exponentes o logaritmos.

A continuación, veremos, de manera resumida, tres casos que ilustran lo dicho a propósito del IG y su virtualidad para los estudios educativos.

\section{Caso primero. Aplicación del IG a las inversiones en Educación Superior}

Eurostat proporciona datos sobre la estructura de la inversión privada (es decir, las aportaciones en los presupuestos familiares) en educación superior por quintiles de ingresos en los diferentes países de la Unión Europea (Eurostat 2019b). Con estos datos, se puede calcular el IG siguiendo el método enunciado anteriormente respecto de líneas quebradas (cálculo del área de trapezoides, etc.). En la Tabla II se ofrecen algunos resultados.

Tabla II. IG de la inversión privada en Educación Superior y de los distintos países

\begin{tabular}{ccc}
\hline ESTADOS EUROPEOS & IG - INV. PRIV. ED. SUPERIOR & IG - BANCO MUNDIAL \\
\hline Austria & 0,664 & 0,374 \\
\hline Bulgaria & 0,709 & 0,374 \\
\hline Chequia & 0,724 & 0,340 \\
\hline Chipre & 0,711 & 0,311 \\
\hline Croacia & 0,708 & 0,265 \\
\hline Eslovaquia & 0,696 & 0,254 \\
\hline Eslovenia & 0,673 & 0,362 \\
\hline España & 0,719 & 0,327 \\
\hline Estonia & 0,660 & 0,271 \\
\hline Finlandia & 0,600 & 0,360 \\
\hline Grecia & 0,726 & 0,304 \\
\hline Hungría & 0,692 & 0,318 \\
\hline Irlanda & 0,673 & 0,342 \\
\hline Letonia & 0,703 & 0,374 \\
\hline Lituania & 0,698 & 0,294 \\
\hline Malta & 0,710 & 0,282 \\
\hline Países Bajos & 0,662 & 0,318 \\
\hline Polonia & 0,705 & 0,355 \\
\hline Portugal & 0,685 & 0,332 \\
\hline Reino Unido & 0,696 & 0,359 \\
\hline Rumanía & 0,723 & \\
\hline
\end{tabular}

Fuente: elaboración propia de Eurostat y Banco Mundial.

Pero además puede establecerse el coeficiente de correlación $(R)$ entre los IG obtenidos a propósito de la inversión educativa en educación superior y los que establece, por ejemplo, el Banco Mundial, para el conjunto de países (Banco Mundial, 2019), que habitualmente proporciona el coeficiente (CG, recuérdese que es el IG por 100), pero que en la tabla anterior hemos puesto como índices (la columna de la 
derecha). En este caso, para el año de referencia, $R=0,433$, que es un valor que indica la tendencia de que la inversión en educación superior sigue el mismo patrón que la desigualdad económica global e incluso la maximiza.

\section{Caso segundo: un Índice de Desajuste en las trayectorias académicas}

En un artículo reciente en prensa hemos formulado un Índice de Desajuste Académico (IDA) para las trayectorias académicas, aplicado a estudios superiores, que permite comparaciones entre diversos instituiciones, centros, grados, años académicos o diferenciación de género, edad, etc. (Diniz et al., 2020, en prensa).

La Tasa de Idoneidad (TI) puede ser definida como el porcentaje de estudiantes de nuevo ingreso en el estudio en un curso que finalizan el estudio inicial en el curso de finalización teórico o antes, y la Tasa de Fluidez (TF) es la «fluidez» en el tránsito del estudiantado por un grado o titulación, definido, formalmente, como el producto entre la idoneidad y la renovación. (Diniz et al., 2020, en prensa). Supongamos un grado o titulación cualquiera, cuya duración teórica es de k cursos. En el año determinado se matriculan unos estudiantes nuevos (que denominaremos $N$ ) respecto del total de los que se matriculan $(M)$. Es trivial que $M \geq N$. La tasa de renovación (TR) es, según la definición habitual y con nuestras siglas: $T R=N / M$. Por otra parte, del conjunto de estudiantes de primer curso (que denominaremos $P$, donde hay una parte de nuevos estudiantes, pero otra de no nuevos, por ejemplo, repetidores), también podemos preguntarnos cuántos de ellos habrán concluido en el año $a+k$, y el número de estos se denominará $Q$. Está claro que $P \geq Q$. Pues bien, la tasa de idoneidad (TI) se define como: $T I=Q / P$. Podemos definir una tasa de fluidez $(T F)$ como: $T F=T R \cdot T I=N Q / M P$.

En este caso se cumplen las condiciones mencionadas anteriormente para el cálculo del IG. Si TR=0, entonces, necesariamente, $M=0$ (o el 0\%), por lo que lógicamente $T F=0$. En segundo lugar, si $T F=1$ (o el 100\%), entonces necesariamente $T R=1$ (y también $T I=1$ ). Por último, dado que $T I \leq 1$, entonces necesariamente $T R \geq T F$, por lo que podemos proceder al cálculo del IG, asignando los valores $T R$ a $\propto$ y $T F$ a y. De esta manera podemos calcular un IG, adaptación que podríamos denominar Índice de Desajuste de Trayectorias Académicas (IDA). Con los datos de la Universidad de València (UV) para la cohorte de entrada de 2013-2014, y para la Universidad Federal de Paraíba (UFPB), para la cohorte 2013.1, obtenemos, por ejemplo, los siguientes resultados con el procedimiento del cálculo mediante curva parabólica:

Tabla III. Índice de Desajuste Académico en la UV y en la UFPB en diversas titulaciones

\begin{tabular}{ccc}
\hline GRADO & IDA-UV & IDA-UFPB \\
\hline Ingeniería & 0,562 & 0,712 \\
\hline Informática & 0,547 & 0,836 \\
\hline Matemáticas & 0,447 & 0,750 \\
\hline Turismo & 0,375 & 0,588 \\
\hline Enfermería & 0,173 & 0,654 \\
\hline
\end{tabular}

Fuente: elaboración propia con los datos académicos.

De lo que se deduce que el desajuste es mayor, en general, en la UFPB que en la UV, y que en ambos casos los mayores ajustes se producen en Enfermería y Turismo, si bien en el caso de la UV se trata del primer grado y en la UFPB el segundo. El mayor desajuste se produce en Ingeniería, en el caso de la UV, y en Informática, en el caso de la UFPB, lo que correspondería, previsiblemente, a mayores proporciones de suspensos. 


\section{Caso tercero: establecimiento de un Índice de Desigualdad en investigaciones sociolingüísticas}

Anteriormente se citó el ejemplo de la tasa de competencia (TK) y la tasa de uso (TU) de una lengua en contextos plurilingües como ejemplo de la atribución de variables $x$ e $y$, en el eventual cálculo del IG. En definitiva, variables de competencia y su realización son frecuentes en el ámbito educativo, y cada vez lo son más con el giro didáctico a las competencias.

En una investigación sobre competencias y usos del catalán en las regiones sociolingüísticas de la Comunitat Valenciana (Hernàndez 2020, en prensa) se han calculado los siguientes IG, con el caso de curvas con un solo valor $\left(x_{i}, y_{i}\right)$, según se consideren curvas parabólicas o exponenciales. Los resultados se presentan en la Tabla IV.

Tabla IV. Índices de Gini aplicados a regiones sociolingüísticas

\begin{tabular}{ccc}
\hline REGIONES SOCIOLINGÜÍSTICAS & IG (PARABÓLICA) & IG (EXPONENCIAL) \\
\hline Alacant & 0,663 & 0,553 \\
\hline Alcoi-Gandia & 0,514 & 0,558 \\
\hline València & 0,414 & 0,465 \\
\hline Castelló & 0,478 & 0,480 \\
\hline Ciudad de València y su área metropolitana & 0,577 & 0,494 \\
\hline
\end{tabular}

Fuente: elaboración propia.

Este caso resulta interesante por cuanto las regiones con menores índices coinciden, pero no aquellas que presentan las puntuaciones mayores en un caso y en el otro. Esto no tiene nada de extraño, por cuanto lo que definen las curvas son, en última instancia, modelos de relación entre dos variables.

\section{Conclusiones y discusión}

En el artículo hemos mostrado la mecánica de cálculo del IG y diversas adaptaciones al ámbito educativo, para evidenciar su capacidad en la investigación sociológica de la educación. Siguiendo este camino, se podrá converger con otras disciplinas que hace décadas impulsaron el cálculo de IG o CG. Esto tiene la ventaja añadida de definir con mayor precisión conceptos habituales en nuestro ámbito, como son los de igualdad y desigualdad. Además, elaborar investigaciones educativas utilizando el IG permite evitar el riesgo de subjetivismo en la elaboración de indicadores compuestos.

Respecto del cálculo en concreto, hemos desentrañado las características principales de la mecánica del cálculo del IG y hemos procedido a su simplificación (entre los muchos métodos utilizables), para permitir fácilmente usos ulteriores. La estimación del IG a partir de trapezoides no presenta ninguna dificultad. Aunque resultan preferibles las estimaciones a partir de ecuaciones de curvas, que son más precisas que los cálculos con líneas quebradas. Se precisan investigaciones ulteriores para calibrar el valor de los modelos subyacentes al uso de curvas parabólicas o exponenciales. Hemos apuntado que hay fórmulas alternativas a la utilizada en el caso de las curvas exponenciales (hemos ofrecido un ejemplo), por lo que en este caso son posibles indagaciones posteriores más clarificadoras. También en el caso de otras curvas, que proporcionarían lógicamente otros modelos de relación de variables.

Sintéticamente, la utilización de indicadores, sea de naturaleza cuantitativa o cualitativa, tiene como objetivo organizar mejor o comprender las informaciones importantes de diferentes elementos que integran el fenómeno observado, entendiendo las complejas relaciones entre variables. Con un buen indica- 
dor se puede medir, de manera sistemática, el desempeño de una institución, por ejemplo, y hacer medidas de intervención, tan pronto se detectan posibles fluctuaciones en un dado proceso, en corto intervalo de tiempo. Por ello, la indagación presentada no solo permite una mejor comprensión de los fenómenos educativos, sino también un debate más clarificador sobre las políticas educativas.

\section{Referencias bibliográficas}

Banco Mundial (2019): Índice de Gini, en https:/ / datos.bancomundial.org/indicador/SI.POV.GINI, consultado el 1 de septiembre de 2019.

Benhamrouche, Aziz y Martín Vide, Javier (2012): “Avances metodológicos en el análisis de la concentración diaria de la precipitación en la España peninsular”. Anales de geografía de la Universidad Complutense, 32(1), 11-27. DOI: https://doi.org/10.5209/rev_AGUC.2012.v32.n1.39306.

Diniz, A.; Villar, Alícia; Hernàndez, F. J. y Castelo Branco, U. (2020): “Índice de desajuste de trayectorias estudiantiles. Caso comparativo de la Universidad Federal de Paraiba y la Universidad de València”. Revista Lusófona de Educaşão

Eurostat (2019a): Income quintile share ratio (S80/S20) by sex. Código tessi180, en: https://ec.europa.eu/eurostat/web/main/home, consultado el 1 de septiembre de 2019.

Eurostat (2019b): Structure of consumption expenditure by income quintile and COICOP consumption purpose. Código: hbs_str_t223, en: https://ec.europa.eu/eurostat/web/main/home, consultado el 2 de septiembre de 2019.

Hernàndez, Francesc J. (2020): "Realitat lingüística valenciana", informe per a la Cätedra de Drets Lingüistics de la Universitat de València. València.

INE (2019): Coeficiente de Gini, en: http:/ / www.ine.es/jaxiT3/Tabla.htm?t=9966, consultado el 2 de septiembre de 2019.

Maldonado, Antonio; Pérez-Ocón, Rafael; Herrera, Amparo (2007): “Depressión and cognition: New insights from the Lorenz curve and the Gini index". International Journal of Clinical and Health Psychology, 7 (1), 21-39.

Martínez Rizo, Felipe. (2012). “Las desigualdades en la educación básica”. Perfiles educativos, 34, 29-46.

OCDE (2011): An overview of growing income inequalities in OECD countries: main findings, en: https:// www.oecd.org/els/soc/49499779.pdf, consultado el 2 de septiembre de 2019.

OCDE (2019): Inequality, en: http://www.oecd.org/social/inequality.htm, consultado el 1 de septiembre de 2019.

Pérez Labajos, Carlos A.; Azofra, Máximo; Blanco Rojo, Beatriz; Achútegui Rodríguez, Juan José: Eguía, Emilio; Díaz, D. (2005): "Collision of Fishing Vessels. Lorenz Curves and GINI Indices". Journal of maritime research, 2(3), 97-106.

Pontriaguin, L. S. (2011). Método de coordenadas. Moscou: Krasand.

Special Study Panel on Education Indicators (1991): Education Counts. An Indicator System to Monitor the Nation's Educational Health. Washington: National Center for Educational Statistics-USA Dpt. of Education. En: http://www.scielo.org.mx/scielo.php?script=sci_nlinks\&ref=7836779\&pid=S1665-10 9X201000020000400022\&lng=es, consultado el 14 de diciembre de 2019. 


\section{Notas biográficas}

Francesc Jesús Hernàndez i Dobon es Profesor de la Universitat de València. Doctor en sociología por la misma universidad y miembro de su Institut de Creativitat i Innovacions Educatives. Su línea de investigación principal es la sociología de la educación.

Uyguaciara Veloso Castelo Branco es Profesora de la Universidad Federal de Paraíba (Brasil). Doctora en historia por la Universidad Federal de Pernambuco, Brasil. En la actualidad realiza un postdoctorado en sociologia en la Universitat de València. Investigadora del Proyecto «Mapa da Educação Superior na Paraíba» (CNPq) y miembro del Grupo de Estudios en Educación Superior y Sociedad (GEES).

Paulo Hideo Nakamura es Profesor jubilado de la Universidad Federal de Paraíba (Brasil). Doctor en ciencias de la educación por la Universidad Autónoma de Asunción (Paraguay). Participa también en el Proyecto «Mapa da Educação Superior na Paraíba» (CNPq) y en el Grupo de Estudios en Educación Superior y Sociedad (GEES). 